FORMATION Formation emploi

Revue française de sciences sociales

135 | Juillet-Septembre 2016

L'économie verte : rupture ou adaptation de la

formation et de l'emploi ?

\title{
Postface : L'emploi dans la transition écologique : des transferts d'emplois massifs pour un impact total faible
}

Rémi Bazillier

\section{CpenEdition}

Journals

Édition électronique

URL : http://journals.openedition.org/formationemploi/4851

DOI : $10.4000 /$ formationemploi.4851

ISSN : 2107-0946

Éditeur

La Documentation française

Édition imprimée

Date de publication : 12 octobre 2016

Pagination : 179-186

ISSN : 0759-6340

Référence électronique

Rémi Bazillier, «Postface : L'emploi dans la transition écologique : des transferts d'emplois massifs pour un impact total faible ", Formation emploi [En ligne], 135 | Juillet-Septembre 2016, mis en ligne le 12 octobre 2016, consulté le 30 octobre 2020. URL : http://journals.openedition.org/formationemploi/ 4851 ; DOI : https://doi.org/10.4000/formationemploi.4851

(c) Tous droits réservés 


\title{
Postface
}

\section{L'emploi dans la transition écologique : des transferts d'emplois massifs pour un impact total faible}

\author{
RÉmI BAZILLIER \\ Professeur en économie. \\ Centre d'Economie de la Sorbonne (UMR CNRS 8174), université 1 Panthéon Sorbonne
}

Ce numéro spécial de la revue Formation Emploi, consacré à l'économie verte, documente et met en avant plusieurs enjeux relatifs au contenu des formations et des emplois liés à la transition écologique. En s'appuyant sur des pratiques dans certains secteurs stratégiques du point de vue du développement durable (bâtiments, transports, agriculture, géosciences,...), les articles soulignent l'importance des changements nécessaires dans le système éducatif et de formation, mais également les difficultés rencontrées par les acteurs devant s'adapter à de nouvelles pratiques et modes de production.

Toutefois, il semble nécessaire de mettre en perspective ces pratiques sectorielles en les replaçant dans le cadre plus large, macroéconomique, des défis liés à l'emploi et aux inégalités dans la transition écologique.

La mise en place de politiques environnementales ambitieuses peut faire l'objet de résistance, notamment du fait des craintes sur les effets de ces politiques sur l'emploi et la compétitivité de l'industrie. La non-prise en compte de ces craintes peut rendre plus difficile le processus de transition écologique, malgré les coûts de mieux en mieux documentés de l'inaction face aux défis du changement climatique, notamment. L'étude des conséquences sociales des politiques visant à améliorer l'environnement est donc primordiale. En France, par exemple, le secteur chimique représente environ 500000 emplois. Comment faire évoluer ces emplois pour prendre en compte la nécessaire protection de l'environnement ? Est-ce que l'émergence de nouveaux " emplois verts " permettra de compenser les possibles destructions d'emplois dans les secteurs polluants amenés à décroître ? Quelles seront les caractéristiques de ces nouveaux emplois, notamment en termes de qualification?

Au niveau international, une lente synergie entre dimension environnementale et dimension sociale du développement durable a émergé au cours des années 1990, quand le Programme des Nations Unies pour l'Environnement (PNUE) a entamé un travail commun avec l'OIT (Organisation internationale du travail). Parmi les différentes initiatives prises conjointement par les deux organisations, notons la publication d'un rapport commun, en 2007, justement sur les « emplois verts » susceptibles d'être créés grâce à la transformation écologique (OIT et PNUE, 2008). 
D'abord, nous reviendrons sur ces coûts de l'inaction face au changement climatique. Nous développerons, dans un second temps, les liens entre transition écologique, politiques environnementales et emplois, avant de discuter plus spécifiquement des coûts et bénéfices de la transition écologique.

\section{Le coût de l'inaction : l'impact du changement climatique sur l'emploi}

Les rapports du GIEC (Groupe intergouvernemental d'experts sur l'évolution du climat) n'ont pas vocation première à mesurer les conséquences sur l'emploi du changement climatique, mais ils analysent tout de même son impact sur les systèmes humains (GIEC, 2014). Cet impact est triple : (1) il affecte directement des activités humaines liées au climat, de l'agriculture au tourisme, (2) il a un impact sur le coût de contrôle des températures internes (hausse du coût de la climatisation et baisse du coût lié au chauffage), (3) il peut également interagir avec d'autres facteurs, par exemple les conséquences relatives aux migrations climatiques.

Le changement climatique peut avoir un effet direct sur l'emploi, par le biais du processus productif, ou indirect via de multiples canaux (Bazillier, 2011). L'impact direct via le processus de production peut facilement être étudié du point de vue théorique, en considérant l'environnement (ou la qualité environnementale) comme un facteur de production. La qualité environnementale pourra alors être considérée comme un stock de capital (naturel), de ressources nécessaires à la production d'un bien donné. Si la qualité environnementale se réduit, la production s'en trouvera négativement affectée. Plusieurs scénarios sont alors possibles : (1) la baisse de la productivité du capital, du travail, ou des deux si le volume de capital ou de travail utilisé reste constant, (2) une baisse de l'utilisation du capital ou du travail ; la baisse de la qualité environnementale se traduisant alors par une perte nette d'emplois, (3) l'utilisation de nouvelles technologies permettant de réduire l'impact environnemental pour une production donnée. L'idée que la technologie puisse être un recours face à un stock de capital naturel déclinant est au cœur du concept de durabilité faible. Selon ce concept, la substitution de la qualité environnementale par la technologie permet un certain développement économique en raison d'un plus grand recours au capital (physique) et au travail. Cette vision de la durabilité faible s'oppose à celle, plus exigeante, de durabilité forte, dans laquelle un système sera considéré comme durable s'il permet de maintenir la qualité environnementale constante.

L'impact sur l'emploi dépendra donc directement de l'impact de la qualité environnementale sur la production, celle-ci ayant un impact sur la consommation. Si le rapport Stern (2007) ne chiffre pas l'impact sur l'emploi, il avance une baisse de la consommation moyenne par individu, comprise entre 5 et $11 \%$, en raison du changement climatique. Cette impact agrégé s'explique par la baisse de la productivité et des rendements des 
activités économiques impactées par le climat, entraînant une baisse du revenu et donc de la consommation. Ceci est susceptible de générer des pertes d'emplois importantes. Rajoutons à cela les conséquences indirectes : impact sur la santé (dépenses de santé et financement de la protection sociale), hausse des coûts de transport et du coût des assurances, liée à la multiplication des catastrophes climatiques, dépenses d'infrastructures,...

Le coût en termes de production, consommation et emploi sera en revanche très hétérogène au niveau mondial. L'impact sera maximal dans les régions les plus pauvres, ce qui est susceptible d'accentuer les inégalités au niveau mondial (Stern, 2007). En Europe, en revanche, rien ne permet de dire que l'impact au niveau agrégé sera négatif. La Confédération européenne des syndicats (2007) propose une analyse exhaustive sur l'Europe des 25 ; basée sur des évaluations sectorielles de l'évolution de la production, elle se concentre sur les secteurs les plus susceptibles d'être touchés par le changement climatique : agriculture, forêt, pèche, tourisme, finance et assurance, et sur les effets directs uniquement (négligeant donc l'impact potentiel sur le prix des actifs et la productivité). Globalement, ce rapport insiste sur les conséquences très différentes entre pays du Sud et pays du Nord de l'Europe ; le changement climatique étant susceptible de favoriser les régions relativement froides et au contraire de défavoriser les régions les plus arides. En Europe, l'impact sur l'emploi sera donc d'abord d'ordre redistributif, des pays du Sud vers les pays du Nord. Or, ces premiers connaissent déjà des taux de chômage plus élevés. Le changement climatique risque donc d'accentuer les inégalités régionales, appelant à de nouvelles solidarités européennes.

\section{Une transition écologique nécessaire pour corriger les « défaillances de marché »}

En dépit de l'accord de la COP $21^{1}$ à Paris, les politiques environnementales sont trop timides pour répondre aux enjeux liés au changement climatique ; cependant, au niveau international, il existe un consensus sur la nécessité de mettre en place des politiques pour enrayer ces évolutions. Celles-ci sont essentiellement de deux types.

On parle de politiques d'adaptation (visant à ajuster les systèmes naturels et humains au changement climatique) et de politiques d'atténuation (ayant pour but de réduire l'impact du changement climatique). L'enjeu actuel est d'assurer la transition d'un modèle économique intensif en ressources vers un nouveau modèle faiblement émetteur de carbone.

Un rapprochement peut être fait avec d'autres grandes révolutions technologiques (la révolution industrielle, les nouvelles technologies,...) ayant entraîné de profonds bou-

1. La COP21 est la 21e conférence des parties à la Convention-cadre des Nations unies sur le changement climatique. Elle a été organisée et présidée par la France, en décembre 2015, et a abouti à l'Accord de Paris, dont l'objectif est de contenir la hausse des températures en deçà de deux degrés. 
leversements. Toutefois, cette transition est rendue nécessaire pour éviter des coûts économiques, écologiques et sociaux de long-terme, contrairement aux autres mutations du système économique qui se sont développées de manière endogène, suite à des innovations ou ruptures technologiques majeures.

L'analogie peut être faite avec le processus de destruction créatrice mis en avant par Joseph Schumpeter dans les années 1940. Le progrès technique ouvre la voie à de nouveaux secteurs d'activités et d'emplois, dont le développement est facilité par la disparition d'activités anciennes, dépassées technologiquement.

Dans un contexte où les externalités environnementales sont nombreuses, nous sommes dans une situation de "défaillance de marché ", qu'il convient de corriger par l'action publique et la coordination des acteurs. L'objectif des politiques environnementales est donc de lancer cette dynamique d'innovations. Les conséquences seront la création de nouvelles activités (ou le verdissement d'activités existantes) et la disparition d'autres, entrainant un processus transitoire qui pourra avoir de multiples répercussions sociales, positives et négatives.

Comme tout processus transitoire, et même si le gain est positif à l'issue du processus, la transition écologique aura des répercussions négatives sur certains groupes sociaux. La Confédération syndicale internationale (CSI) et l'OIT ont donc concentré leur attention sur la nécessité d'une transition juste, protégeant les plus faibles et associant la justice sociale à la mutation écologique. Elle se fonde sur plusieurs principes : (i) la création de vraies perspectives vertes et des investissements durables de long terme, (ii) une évaluation précoce des effets sur l'emploi, (iii) le dialogue social pour associer l'ensemble des parties prenantes au changement, (iv) la formation et le renforcement des systèmes d'éducation, (v) la protection sociale pour faire face à la vulnérabilité environnementale.

Parmi les différents instruments de lutte contre la pollution, les politiques de taxation et de quotas (mécanismes de droits à polluer) consistent à fixer un prix à la pollution afin de rendre celle-ci coûteuse pour les entreprises qui la génèrent. Ces politiques sont porteuses d'effets redistributifs importants : en augmentant les coûts, elles peuvent inciter les entreprises à licencier pour maintenir les niveaux de rentabilité. Mais les revenus générés par une taxe écologique permettront aussi à l'Etat d'assurer des recettes fiscales supplémentaires. L'utilisation d'un tel revenu pourra servir à atteindre d'autres objectifs, notamment la baisse du chômage. C'est ce qu'on appelle le double-dividende. La taxe serait donc un moyen de réduire la pollution (premier dividende) et d'améliorer la situation de l'emploi (second dividende). L'impact final sur l'emploi dépendra donc de l'effet négatif dont elle est la cause directe, qu'il faut comparer avec l'impact positif que pourrait provoquer une baisse de la taxation sur le travail par exemple. Malheureusement, il apparaît que l'existence d'un effet positif net est largement discutable et dépendra d'une série de conditions restrictives (Bovenberg, 1995). Les études empiriques mettent toutefois en avant un effet moyen très légèrement positif sur l'emploi. Patuelli et al (2005) réalisent une méta-analyse 
sur les effets de la taxation écologique et trouvent un effet positif de l'ordre de $0.5 \%$ de l'emploi total.

Il convient également de prendre en compte le caractère potentiellement anti-redistributif d'une telle taxe, qui touchera plus fortement les ménages les plus pauvres. Les dépenses en énergie pèsent plus fortement dans leur budget et ils seront donc plus affectés financièrement. Les revenus liés à la taxe pourraient être redistribués de manière à corriger les effets négatifs sur les inégalités (Schubert, 2010). Mais dans ce cas, les ressources fiscales générées par la taxe ne pourront viser à améliorer l'emploi.

\section{Des transferts d'emplois massifs induisant des enjeux 3 en termes de formation}

Quelle que soit la politique environnementale mobilisée pour enclencher ce processus de transition écologique, celle-ci est donc susceptible de provoquer des coûts à court terme, pouvant être éventuellement compensés par des bénéfices sur le plus long terme. Les coûts sont de multiples natures : (1) la demande pour les biens polluants est appelé à baisser du fait de l'augmentation de leur prix, (2) les gains d'efficacité énergétique nécessitent des investissements pouvant être couteux à court-terme, (3) les technologies faiblement intensives en carbone peuvent être plus onéreuses que celles utilisant des ressources fossiles.

Mais de nouvelles opportunités de marché susceptibles de créer des emplois peuvent surgir. C'est le pari du développement des " emplois verts " auxquels ce numéro de la revue Formation Emploi est consacré. L'hypothèse de Porter (Porter et al., 1995)permet de comprendre comment ce phénomène est susceptible d'apparaître. Il s'agit d'une amélioration de la productivité résultant d'une législation environnementale plus contraignante. Un renforcement de la réglementation est ainsi susceptible d'entraîner des coûts supplémentaires pour les entreprises. Mais pour faire face à ces coûts additionnels, les entreprises peuvent être incitées à améliorer leur productivité. Elles peuvent également profiter des opportunités offertes par la mutation écologique. Dans cette perspective, les politiques environnementales sont susceptibles d'être à l'origine d'une vague d'innovations environnementales favorables à l'emploi (Porter et al., ibid.). C'est le moteur de la croissance verte que de nombreux décideurs visent à promouvoir.

L'OIT et le PNUE (2007) ont cherché à estimer le nombre d'emplois pouvant résulter de ces innovations environnementales, et plus largement de la transition vers une économie faiblement intensive en carbone. Ce rapport marque une étape importante dans la conciliation des enjeux sociaux et environnementaux, dans la mesure où il s'agit à la fois de créer un grand nombre "d'emplois verts " mais également de s'assurer que ces emplois sont de qualité et respectent les critères de travail décent définis par l'OIT. Selon ce rapport, la mutation écologique a quatre conséquences principales sur l'emploi : (i) des créations d'emplois vont être nécessaires pour produire de nouveaux biens et équipements, 
(ii) des emplois vont être substitués à d'autres (transfert d'énergies fossiles vers les énergies renouvelables), (iii) des emplois vont être supprimés sans remplacement direct, (iv) beaucoup d'emplois vont évoluer du fait des exigences environnementales. Cela entraînera une modification des compétences requises ou des modes de travail.

Selon ce rapport, plusieurs millions d'emplois verts existent d'ores et déjà dans le monde : 2,3 millions dans les énergies renouvelables ou 4 millions dans l'efficacité énergétique. Mais ces emplois sont aujourd'hui largement concentrés dans certains pays (essentiellement des pays développés) et dans certains secteurs. Beaucoup d'emplois existants offrent par ailleurs de mauvaises conditions de travail ou sont mal rémunérés (dans le secteur du recyclage par exemple). Le nombre de ces emplois est amené à exploser au cours des prochaines années. L'OIT et le PNUE chiffrent ainsi à 2,5/ 3 millions les créations d'emplois verts aux Etats-Unis et en Europe, et le potentiel est encore plus important pour les pays en développement. Dans le secteur des énergies renouvelables, le potentiel de création d'emplois excéderait les 20 millions, soit un nombre d'emplois supérieur à celui de l'industrie des énergies fossiles.

En Europe, la CES (2007) estime que l'effet global sur l'emploi d'une réduction des émissions de $\mathrm{CO} 2$ de $30 \%$, d'ici à 2030, serait légèrement positif, de l'ordre de $1.5 \%$ (par rapport à une situation dans laquelle les émissions de $\mathrm{CO} 2$ continueraient à croître de $1 \%$ par an). Si l'impact global est relativement faible, le rapport prévoit des transferts d'emplois massifs, essentiellement au sein même de secteurs. Les entreprises innovantes embaucheront massivement, tandis que celles ne réagissant pas devront licencier. Quant aux évolutions intersectorielles, on peut prévoir un transfert des emplois liés à la production d'énergie vers des emplois ayant trait à l'amélioration de l'efficacité énergétique, un transfert des emplois par la route vers des emplois dans les transports publics et le fret, et enfin un transfert des emplois d'ingénieurs, designers, commerciaux, etc., des secteurs de l'industrie liée à l'énergie fossile vers l'industrie des énergies renouvelables.

Globalement, ces nouveaux emplois seront plus qualifiés que les emplois détruits. La mutation écologique crée donc de nouveaux défis en termes de formation de la maind'œuvre et de systèmes de protection sociale pour les travailleurs les moins éduqués. Les articles de ce numéro spécial insistent largement sur les enjeux sectoriels, à la fois en termes de formation initiale (avec l'exemple du secteur des géosciences) que de formation professionnelle (dans les énergies renouvelables, le transport, le bâtiment).

En France, plusieurs études avaient chiffré les effets du Grenelle de l'environnement ${ }^{2}$. Le chiffre de 500000 emplois avait été avancé par le ministre de l'Environnement de l'époque, Jean-Louis Borloo. Le Boston Consulting Group prévoyait la création de 60000

2. Le Grenelle de l'environnement fait référence à une série de réunions ayant eu lieu en 2007 et rassemblant tous les acteurs concernés par les questions environnementales. Il a débouché sur l'adoption de deux lois, Grenelle I et Grenelle II. Ces lois regroupent une série de mesures visant à favoriser la transition écologique dans le domaine de l'énergie, de l'agriculture, du bâtiment et des transports notamment. 
emplois et le WWF 635 000. Toutefois, ces estimations optimistes avaient été remises en cause par une étude de la direction générale du Trésor (Briard et al., 2010), qui prévoyait une baisse de l'emploi et de la croissance après 2020, une fois les investissements réalisés et en raison de la persistance des coûts additionnels. En effet, les investissements (constructions de lignes de tramways par exemple) ont des effets bénéfiques principalement au moment où ils sont réalisés, leur efficacité sur le long terme dépendant de leurs rendements. En revanche, les coûts liés à la mise en place d'une fiscalité écologique ou de nouvelles normes environnementales sont permanents, ce qui peut expliquer ces moindres effets. En 2023, on aboutirait à un solde net négatif de 120000 emplois. Mais ces conclusions ont fait l'objet de critiques, notamment parce que le modèle ne prend pas en compte les coûts plus importants liés à l'inaction et les gains relatifs à la spécialisation dans des secteurs plus dynamiques.

\section{Conclusion}

Ce numéro spécial de la revue Formation Emploi s'attache à comprendre les enjeux, en termes d'emploi et de qualification, liés à l'économie verte. À partir de plusieurs cas sectoriels, l'adaptation des systèmes éducatifs et de formation apparaît comme une condition nécessaire à la réussite de la transition écologique. Il faudra à la fois former plus d'étudiants dans les secteurs amenés à se développer en raison de cette transition (voir l'exemple des géosciences), mais également adapter la formation et la qualification d'emplois existants. Plusieurs articles de ce numéro soulignent les difficultés concrètes pouvant être rencontrées dans la modification des pratiques des acteurs, mais également celles dues aux hésitations ou ambiguïtés des pouvoirs publics.

Dans cette postface, nous insistons sur les enjeux macroéconomiques liés à la transition écologique qui permettent de saisir l'ampleur des défis. Si l'effet agrégé sur l'emploi sera sans doute faible (mais significatif), les transferts d'emplois seront massifs, tant à l'intérieur des secteurs qu'entre secteurs. Ce panorama global renforce l'idée que les systèmes éducatif et de formation professionnelle devront prendre en compte ces nouveaux défis.

Le dernier enjeu essentiel est celui des inégalités. Comme nous l'avons évoqué, la hausse des dépenses énergétiques pèse plus lourdement sur les plus pauvres et les nouveaux emplois seront en moyenne plus qualifiés que les emplois détruits. Le système de protection sociale devra prendre en compte ces évolutions afin, notamment, de lutter contre les phénomènes de précarité énergétique et d'exclusion du marché du travail pour les salariés non formés.

La dimension sociale de la transition écologique est donc essentielle et peut même conditionner l'acceptabilité des politiques environnementales. Si les politiques publiques négligent cette dimension, les oppositions risquent de mettre en péril la réussite de ce processus de transformation de l'économie. Comme le suggèrent Crifo et al. (2012) dans leur ouvrage propositionnel sur l'économie verte, il semble nécessaire à la fois de proposer 
une "politique social-écologique " et de s'assurer que la fiscalité soit juste, malgré le caractère potentiellement anti-redistributif des politiques environnementales. Les politiques de formation et d'emploi devront également prendre en compte ces nouveaux enjeux pour éviter que la transition écologique devienne un nouveau facteur d'exclusion. Ainsi, la transition écologique est peu susceptible d'avoir un impact agrégé important sur l'emploi ; cependant, elle implique des transferts massifs d'emplois qui devront être accompagnés par une politique pertinente en la matière.

\section{Bibliographie}

Bazillier R. (2011), Le travail, grand oublié du développement durable, Paris, Le Cavalier Bleu, col. « eDDen ", 222 p.

Briard P., Fery P., Galko E., Guillerminet M. L., Klein C. \& Ollivier T. (2010), « Impacts macroéconomiques du Grenelle de l'Environnement ", Les Cahiers de la DG Trésor, $\mathrm{n}^{\circ}$ 2010/06, décembre.

Bovenberg A. L. (1995), "Environmental taxation and employment", De Economist, 143(2), pp. 111-140.

CES (2007), Climate Change and Employment : Impact of Employment in the European Union-25 of climate change and CO2 emission reduction measures by 2030, Confédération européenne des syndicats, en partenariat avec ISTATS, SDA, SYNDEX et Wuppertal Institute.

Crifo P., Glachant M., Hallegatte S., Eloi L., Gérard R. (2012), L'économie verte contre la crise : 30 propositions pour une France plus soutenable, Paris, PUF.

GIEC (2014), Cinquième rapport d'évaluation du GIEC : Changements climatiques, disponible ici : https://www.ipcc.ch/home_languages_main_french.shtml\#tabs-3

OIT \& PNUE (2008), Green Jobs: Towards Decent Work in a Sustainable, Low-carbon World, september.

Patuelli R., Nijkamp P. \& Pels E. (2005), "Environmental tax reform and the double dividend: A meta-analytical performance assessment”, Ecological Economics, 55(4), pp. 564-583.

Porter M. E. \& Van der Linde C. (1995), "Toward a new conception of the environmentcompetitiveness relationship", The journal of economic perspectives, 9(4), pp. 97-118.

Schubert K. (2010), Pour la taxe carbone. La politique économique face à la menace climatique, Opuscule CEPREMAP, Paris, Editions rue d'Ulm.

Stern N. (2007), The economics of climate change: the Stern report, Cambridge, UK. 